\title{
Cooling a Spin Relaxation Hot Spot
}

\section{The rate at which electron spins relax in silicon quantum dots is controlled by the strength and direction of external magnetic fields.}

By Marric Stephens

lectron spins in silicon quantum dots (SQDs) could one day represent the 0 's and 1's in quantum computers-but only if they can last long enough to be measured. The rate at which these spin states decay depends on perturbations to the material's electronic band structure by local electric and magnetic fields. Certain field configurations are known to enhance this decay-an unwanted effect for quantum technologies. Now, Xin Zhang and Rui-Zi Hu, at the University of Science and Technology of China, and colleagues demonstrate a method to instead suppress this undesirable SQD behavior using a magnetic field.

In their experiments, the team applied a magnetic field to an SQD and then measured its electron-spin decay rate as they changed the field's orientation relative to one of silicon's crystal planes. For a 0.8-T field they detected a two order-of-magnitude variation in the decay rate, with the maximum occurring when the field was angled at $9^{\circ}$ to the crystal plane. For a 1.5-T field, however, they detected only a

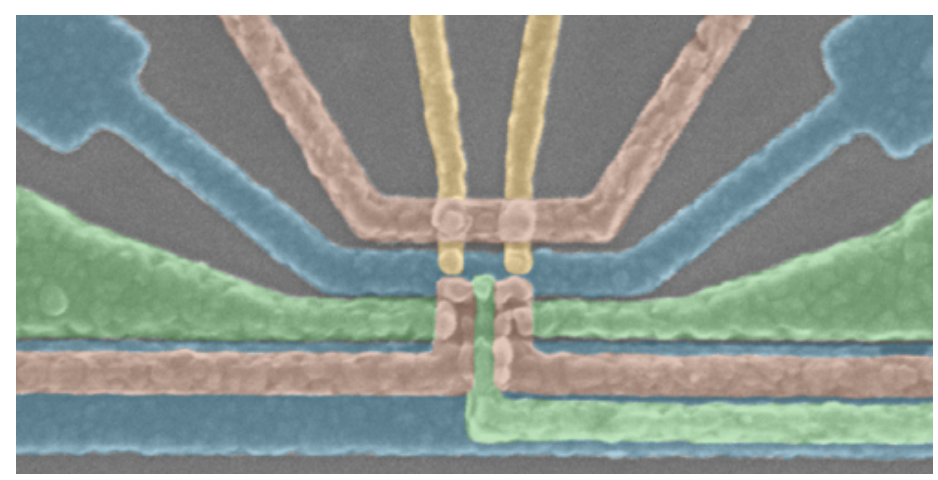

factor-of- 6 variation in the spin decay rate.

The researchers attribute the decrease in variability to the coupling and mixing of certain electronics states. The spin of an excited electron can only flip its spin direction-from spin up to spin down, for example-if it can shed its excess energy. In an SQD this change requires that the electron move to another orbital state or another valley state (a state associated with the dip in a particular electronic band.) The magnetic field they apply affects the energetic accessibility of these transition paths, increasing or decreasing the spin decay rate depending on the field's strength and orientation. Zhang, $\mathrm{Hu}$, and colleagues say that the effect hints at ways to optimize spin lifetimes for quantum-computing applications.

This research is published in Physical Review Letters.

Marric Stephens is a Corresponding Editor for Physics based in Bristol, UK. 